\title{
Minimizing data collection for field calibration of steady-state virtual sensors for HVAC equipment
}

\author{
Howard Cheung* ${ }^{1}$, James E. Braun ${ }^{2}$ \\ ${ }^{1}$ The Hong Kong Polytechnic University \\ ${ }^{2}$ Ray W. Herrick Laboratories, Purdue University \\ *corresponding author (email: howard.at@gmail.com; Tel: +852 2766 5994; Fax: +852 2765 \\ 7198)
}

\section{Keywords}

Virtual sensor, Accuracy, Uncertainty, Applicability, Design of experiments

\begin{abstract}
The paper presents an algorithm to minimize the amount of data collection for calibration of steady-state virtual sensors when obtained during normal operation of HVAC equipment in the field. If virtual sensors were calibrated using laboratory data, the amount of the calibration data could be minimized using design of experiment techniques. However, these techniques are not applicable to data from field operating equipment since most of the operating conditions are not controllable (e.g., ambient temperature, loads). In this paper, an algorithm to minimize the data collection period for calibration of steady-state virtual sensors is developed based on sensor accuracy, reliability and applicability. Application of the method is demonstrated for calibration of hourly electricity consumption virtual sensors for 3 packaged air conditioners that are serving a small commercial building, and the method terminated the calibration process after about 3 weeks of data collection for all units with very accurate estimates of electricity consumption.
\end{abstract}

\section{Nomenclature}

$\begin{array}{ll}c_{p} & \text { Constant pressure specific heat }\left[\mathrm{kJ} \mathrm{kg}^{-1} \mathrm{~K}^{-1}\right] \\ c_{v} & \text { Constant volume specific heat }\left[\mathrm{kJ} \mathrm{kg}^{-1} \mathrm{~K}^{-1}\right] \\ \Delta \hat{y} & \text { Uncertainty of virtual sensor that estimates variable y [varies] } \\ \text { cov } & \text { Coefficient of variation } \\ E & \text { Electricity consumption }[\mathrm{kWh}] \\ \boldsymbol{H} & \text { Hessian matrix [varies] } \\ \boldsymbol{I} & \text { Operating status }\end{array}$




\begin{tabular}{|c|c|}
\hline Ind & Convergence indicator [varies] \\
\hline $\boldsymbol{J}$ & Jacobian matrix [varies] \\
\hline$m$ & Number of coefficients \\
\hline $\mathrm{n}$ & Number of data points \\
\hline$N$ & Number of data points within an hour \\
\hline$N_{h}$ & Number of hours within the calibration period \\
\hline$P$ & Pressure $[\mathrm{kPa}]$ \\
\hline$T$ & Temperature $[\mathrm{K}]$ \\
\hline$t_{D O F, 1-\alpha}$ & $\begin{array}{l}t \text { statistics of student } t \text { distribution under a confidence interval } 100(1-\alpha) \% \\
\text { and a degree of freedom DOF }\end{array}$ \\
\hline$\dot{W}$ & Power consumption $[\mathrm{kW}]$ \\
\hline$\vec{x}$ & Input vector [varies] \\
\hline$x_{i j}$ & The entry on the $\mathrm{i}^{\text {th }}$ row and $\mathrm{j}^{\text {th }}$ column of matrix $\mathbf{X}$ \\
\hline$\vec{y}$ & Output vector [varies] \\
\hline$\hat{y}$ & Predicted value of variable y by a virtual sensor [varies] \\
\hline $\bar{y}$ & Mean of variable $z$ [varies] \\
\hline \multicolumn{2}{|l|}{ Greek } \\
\hline$\beta$ & Coefficients [varies] \\
\hline$\Delta x$ & Uncertainty of variable $\mathrm{x}$ [varies] \\
\hline$\kappa$ & Specific heat ratio \\
\hline$\sigma_{x}$ & Sample standard deviation of variable $x$ [varies] \\
\hline$\sigma_{y x}$ & Mean square error of predicted variable of $y$ at input $x$ [varies] \\
\hline$\tau$ & Time [hour] \\
\hline \multicolumn{2}{|l|}{ Subscript } \\
\hline acc & accuracy \\
\hline app & applicability \\
\hline cal & calibration data \\
\hline comp+cond & compressor and condenser fan \\
\hline cond & condenser return bend \\
\hline current & current data acquisition period \\
\hline evap & evaporator inlet/ evaporator fan \\
\hline previous & previous data acquisition period \\
\hline rel & reliability \\
\hline test & user-defined test case \\
\hline v & saturated vapor \\
\hline
\end{tabular}

\section{Introduction}

Virtual sensors reduce the cost to measure equipment states or other performance indices by using models (e.g., regression equations) that relate the desired outputs to low cost 
measurements. In recent years, there have been a number of developments of steady-state virtual sensors for different building applications. Li et al. (2011) has given an extensive literature review on the development of virtual sensors in building systems that includes virtual sensors for refrigerant pressure, refrigerant mass flow rate, capacity of cooling coils, etc. Kusiak et al. (2010) used neural network models to estimate temperature, relative humidity and carbon dioxide concentration in an indoor environment based on outdoor conditions. Yu et al. (2011) presented a virtual sensor for airflows in packaged air conditioners. Zhao et al. (2012) developed a condenser fouling sensor for water-cooled chillers. Song et al. (2013) developed a virtual sensor for water flow in an air-handling unit that is based on a water valve characterization. Wang et al. (2014) presented a virtual sensor for airflow in air handling units. Kim and Braun (2015) evaluated a virtual sensor that estimates the charge inventory in variable-speed vapor compression systems based on superheat and subcooling measurements. Cheung and Braun (2015) developed virtual sensors for power consumption in packaged air conditioners. Yu and Li (2015) used a supply air temperature virtual sensor for packaged air conditioners to demonstrate a calibration scheme for degraded sensors using field operating equipment data.

Virtual sensors can be calibrated using either laboratory or field data. Although more calibration data usually leads to sensors with higher accuracy, reliability and applicability, the cost of collecting calibration data increases with the length of the collection period because the data acquisition equipment needed for the calibration is not permanently installed. For laboratory testing, the amount of calibration data can be minimized by controlling the operating conditions using design of experiments techniques. These include the Taguchi method (Taguchi et al. 2005), Latin Hypercube Sampling (Mckay et al. 2000), D-optimality method (John and Draper 1975), or other techniques that are defined in design of experiment textbooks (Mongomery 2004; Goos and Jones 2011). One example of using design of experiments in virtual sensor development can be found in Vasudevan (2015), where the D-optimality method was used to reduce the size of the laboratory data set needed for calibration of a virtual refrigerant charge sensor by $50 \%$ without significant loss in accuracy.

However, these techniques are not applicable to obtain calibration data for equipment that is operating in the field because the operating conditions cannot be fully controlled. As a result, the length of time necessary for collecting calibration data to obtain accurate virtual sensors is significantly greater than that for laboratory testing. The data needed for calibration typically includes expensive measurements (e.g., power) that cannot be left in place. Otherwise, there would be no need for the virtual sensors. One step to reduce the data acquisition period with uncontrollable operating conditions is to understand the effect of the amount of calibration data on the calibration result. Song et al. (2013) and Yu and Li (2015) considered the effect of the number of data points on the magnitude of the uncertainty of the virtual sensors according to the Student $t$ distribution but did not consider the effect of operating conditions within the calibration data set on sensor applicability. Reddy and Claridge et al. (2000) effectively studied the effect of data on regression equation applicability by calculating the uncertainty of energy savings from building simulations based on the correlation of the regression data and the error 
of regression equations. Cheung and Braun (2015) showed that the choice of calibration data can affect the uncertainty of virtual sensor outputs. Despite their study of the effects, they did not include any application of the studies to minimize the amount of calibration data.

In the current paper, an algorithm is presented that can automatically terminate the data acquisition period based on sensor accuracy, reliability and applicability. The method has been applied for calibration of a steady-state virtual sensor for hourly electricity consumption virtual sensors. Results are presented for 3 different packaged air conditioners that are operating at a small commercial building.

\section{Algorithm development}

Calibration of virtual sensors is essentially a parameter estimation problem that involves linear or non-linear regression applied to a set of calibration data. The algorithm for minimizing the amount of data for virtual sensor calibration analyzes outputs from a reoccurring regression process, and it stops data acquisition when any one of the following three criteria is satisfied:

1. the user requirements for sensor performance in terms of accuracy, reliability and applicability are met;

2. extra data is unlikely to improve the sensor performance;

3. the collection period has exceeded an upper limit.

If none of the criteria are met, then the algorithm continues to acquire data and repeat the regression process. The second and third criteria are meant to ensure an upper limit to the cost for calibrating the model and are similar to termination criteria on the maximum number of iterations in optimization algorithms (Nocedal and Wright 2006). A flowchart for the algorithm is shown in Figure 1. The virtual sensor calibration and termination algorithm are successively applied after "short" data acquisition intervals (e.g., 2 days) defined by the user. 


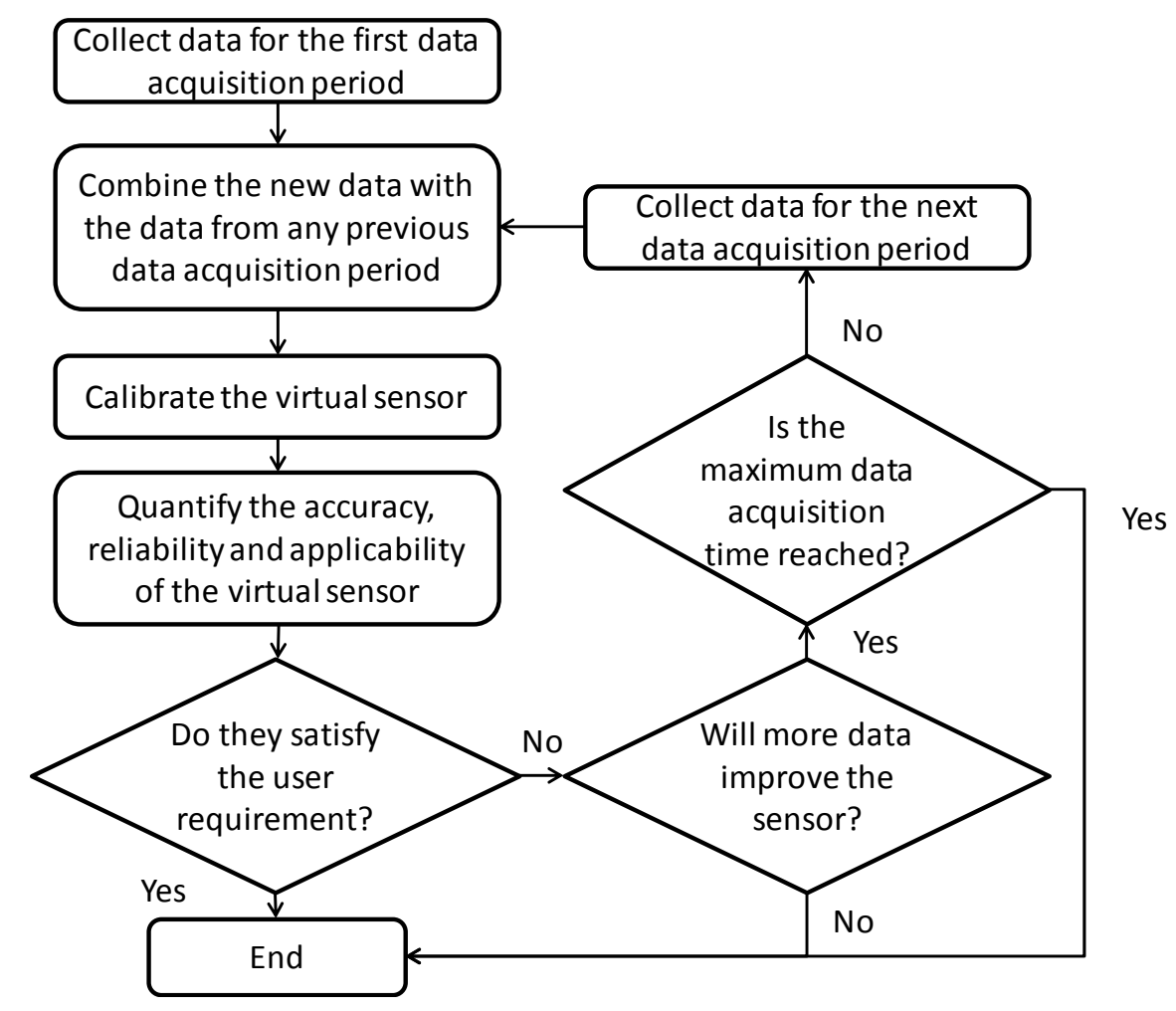

Figure 1 Flowchart illustrating the operation of the minimization algorithm

Figure 1 does not include any active control override of the HVAC equipment during the data collection because some inputs to HVAC equipment virtual sensors are weather dependent and uncontrollable and the calibration process is designed not to interrupt the building occupants served by the HVAC equipment during the data collection process.

To determine if the data acquisition should end according to Figure 1, the following features are calculated from the calibration results of the virtual sensor.

- The performance indicators for accuracy, reliability and applicability of the virtual sensor

- The convergence criteria to estimate if more data would improve the sensor

The accuracy of a virtual sensor in this algorithm is defined by the coefficient of variation in Eqn.(1).

$$
\operatorname{cov}=\sqrt{\frac{\sum_{i}\left(y_{c a l, i}-\hat{y}_{i}\right)^{2}}{n_{c a l}-1}} \frac{n_{c a l}}{\sum_{i} y_{c a l, i}}
$$

Coefficient of variation is widely used to evaluate accuracy of regression results. For instance, ASHRAE Guideline 14 (ASHRAE 2014) demonstrates its use in evaluating the accuracy of measurement and verification methods for building energy savings. A small coefficient of 
variation suggests that the virtual sensor predicts the system operation in the calibration scenarios accurately.

The reliability of a virtual sensor at any stage in the calibration process depicted in Figure 1 is quantified by the average uncertainty of the virtual sensor output over all of the current calibration data using Eqn. (2).

$$
\overline{\Delta \hat{y}}_{c a l}=\frac{1}{n_{c a l}} \sum_{i} \Delta \hat{y}\left(\vec{x}_{c a l, i}\right)
$$

where $\mathrm{i}$ is an index for the calibration input data from 1 to the end of the current available data and $\mathrm{n}_{\text {cal }}$ is the current number of calibration data points. The average uncertainty in Eqn. (2) is an estimate of the virtual sensor output uncertainty if the calibration process were repeated. $A$ small value of average uncertainty means that the estimates from the virtual sensor are reliable. A general method for calculating the uncertainty of virtual sensor outputs was developed by Cheung and Braun (2015) and is used to calculate the uncertainty with respect to the calibration process. In this method, the uncertainty of the virtual sensor output is decoupled into different components related to regression model uncertainty, uncertainty in data measurements used for model calibration, uncertainty in virtual sensor input measurements and the uncertainty in the measured outputs. Other methods of uncertainty calculation of virtual sensor outputs can also be applied to calculate the average uncertainty in Eqn. (2).

The virtual sensor output uncertainty determined with Eqn. (2) for a given calibration data set is limited because the range of inputs encountered in the calibration data set may not be reflective of the entire range that may be experienced in the future for this application. Therefore, the applicability of the virtual sensor is quantified by the average uncertainty of the virtual sensor outputs for a user-defined test case determined using Eqn. (3).

$$
\bar{\Delta}_{\text {test }}=\frac{1}{n_{\text {test }}} \sum_{i} \Delta \hat{y}\left(\vec{x}_{\text {test }, i}\right)
$$

The user-defined test case specifies a data set of inputs for evaluating the current virtual sensor uncertainties using methods that quantify extrapolation uncertainty appropriately and should be reflective of the expected range for those inputs. If the sensors work well over the application range, Eqn. (3) should give a small value. Since the uncertainty calculated using the Cheung and Braun (2015) method predicts larger uncertainty for extrapolation, the method is used to calculate the uncertainties in Eqn. (3).

When the performance indicators in Eqns.(1), (2) and (3) are all lower than some user-defined thresholds, the virtual sensor satisfies the user requirements on its accuracy, reliability and applicability. This implies that there are sufficient calibration data to fit the user's needs, and the collection of calibration data can be stopped according to criterion 1 in the list in the beginning of this section. 
To determine if extra data cannot improve sensor performance and data collection should be stopped according to criterion 2, convergence indicators are evaluated. They are calculated based on the performance indicators in Eqn.(1), Eqn. (2), and Eqn. (3). The resultant equations for convergence indicators are Eqns. (4), (5) and (6).

$$
\begin{aligned}
\operatorname{Ind}_{\mathrm{acc}} & =\left|\frac{\operatorname{cov}_{\text {cal,current }}-\operatorname{cov}_{\text {cal,previous }}}{\tau_{\text {cal,current }}-\tau_{\text {cal,previous }}}\right| \\
\operatorname{Ind}_{\mathrm{rel}} & =\left|\frac{\overline{\mathrm{y}}_{\text {cal,current }}-\bar{\Delta}_{\text {cal,previous }}}{\tau_{\text {cal,current }}-\tau_{\text {cal,previous }}}\right| \\
\operatorname{Ind}_{\mathrm{app}} & =\left|\frac{\bar{\Delta}_{\text {test,current }}-\bar{\Delta}_{\text {test }, \text { previous }}}{\tau_{\text {cal,current }}-\tau_{\text {cal,previous }}}\right|
\end{aligned}
$$

Eqn. (4) checks the convergence of the accuracy of a virtual sensor, and a small value implies that the accuracy of the virtual sensor is not likely to be improved by more calibration data. Eqn. (5) examines the convergence of the reliability of a virtual sensor, and a small value implies that the virtual sensor is unlikely to become more reliable by collecting more calibration data. The last convergence indicator in Eqn. (6) determines if the applicability of the virtual sensor improves with more calibration data, and a small value implies that collecting more calibration data is not likely to improve the applicability of the sensor.

When all three convergence indicators are smaller than some user-defined thresholds, it is expected that more data would not significantly change the sensor accuracy, reliability and applicability. This means that further data collection is unnecessary and the data acquisition should be stopped even if the virtual sensor cannot satisfy the user requirement.

Lastly, it is possible to collect calibration data that improve sensor performance slowly and can only satisfy the user requirement after a long and costly data acquisition period. In this case, the convergence indicators are always higher than the user-defined thresholds, and the performance indicators only fall below the thresholds after a data acquisition period that is too costly for the user. To avoid this from happening, the data acquisition should also be stopped after a maximum data acquisition period regardless of the values of performance and convergence indicators, and the algorithm stops the data acquisition by criterion 3 .

The calibration termination algorithm requires the following four user-defined parameters:

- length of short data acquisition period between each update of virtual sensor calibration;

- test case for applicability assessment;

- thresholds to stop the minimization algorithm;

- maximum length of data acquisition period.

The selection of these parameters depends on the cost of data acquisition per day, the expected operating conditions of the equipment and the computational power available for calibration. 


\section{Description of the case study}

\section{Experimental setup}

The case study involves 3 single-speed packaged air conditioners operating in Philadelphia, Pennsylvania in the U.S. in August and September of 2013 as described by Cheung and Braun (2015). Some specifications associated with the units appear in Table 1.

Table 1 Specifications of experimental setups

\begin{tabular}{|l|l|l|l|}
\hline Packaged air conditioners & RTU 2 & RTU 3 & RTU 4 \\
\hline Nominal cooling capacity [kW] & 14.1 & 14.1 & 17.2 \\
\hline Refrigerant & R22 & R22 & R410A \\
\hline Compressor & Reciprocating & Reciprocating & Scroll \\
\hline Condenser airflow [cfm] & 4000 & 4000 & 4000 \\
\hline
\end{tabular}

Measurements were installed on the units to facilitate calibration and implementation of virtual power consumption sensors described in the next section. A schematic of the experimental setup is shown in Figure 2. In Figure 2, T represents surface mounted thermocouples to measure refrigerant temperatures, $\mathrm{S}$ represents current switches installed the power supply of the evaporator fans and compressor to indicate if they were operating and $W$ represents an energy meter. The condenser thermocouple was installed on the surface of the return bend of the condenser coil in order to provide a representative value for the condensing temperature. The inlet to the evaporator coil measures the evaporation temperature. These temperatures are inputs to the virtual power sensors along with compressor inlet temperature. T-type thermocouples with uncertainty of $\pm 0.5 \mathrm{~K}$ were used for the surface mounted temperature measurements.

Only one current switch was needed for the compressor and condenser fan because they operate simultaneously. The electricity consumption of the air conditioning unit was measured using energy meters that gave a reading for every $0.1 \mathrm{kWh}$ of electricity consumed, and the hourly electricity consumption was calculated by counting the number of readings in the hour by Eqn. (7)

$$
E=\text { Number of readings of } 0.1 \mathrm{kWh} \text { within an hour } \mathrm{x} 0.1 \mathrm{kWh}
$$

Because the increments of $E$ in Eqn. (7) were $0.1 \mathrm{kWh}$, the uncertainty of E calculated by Eqn. (7) was assumed to be $\pm 0.05 \mathrm{kWh}$. A data acquisition system transmitted the data to a server every minute for the hourly electricity consumption virtual sensors. 


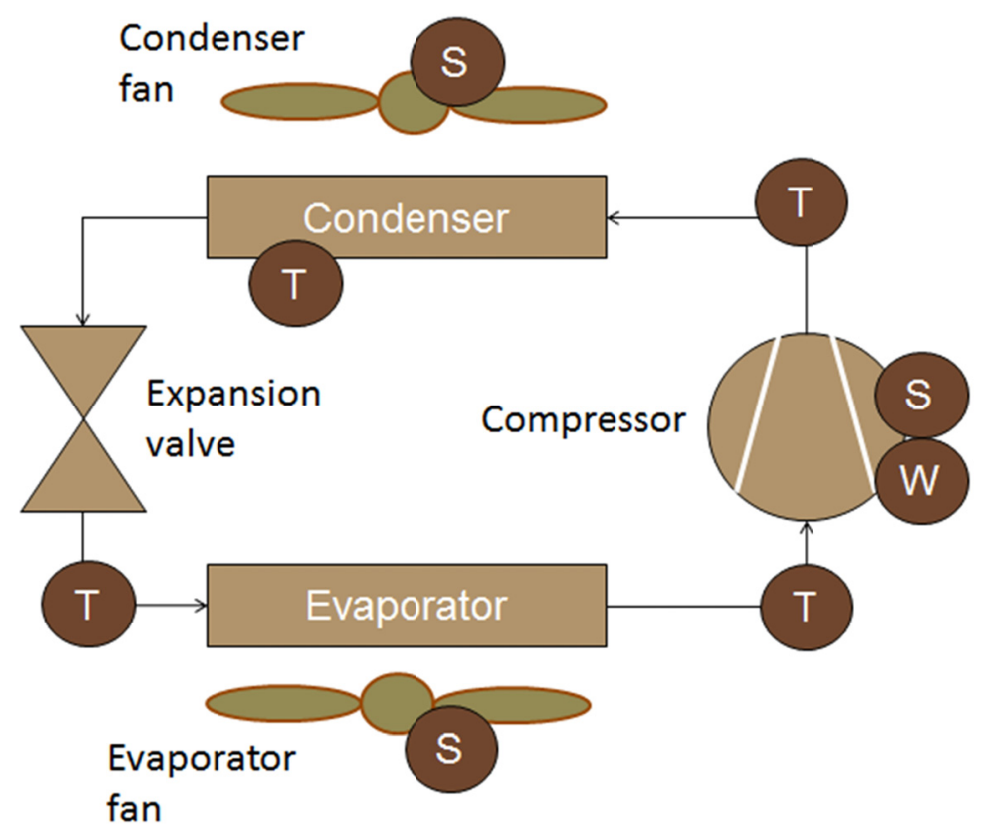

Figure 2 Schematic of sensor installation in each RTU (Cheung and Braun (2015))

\section{Hourly electricity consumption virtual sensor of a packaged air}

\section{conditioner}

A virtual sensor to estimate the hourly electricity consumption of the packaged air conditioners was developed based on two power consumption virtual sensors presented by Cheung and Braun (2015): 1) compressor and condenser fan power and 2) evaporator fan power.

The two power consumption sensors were used to estimate the total power consumption of a packaged air conditioner as Eqn. (8).

$$
\widehat{\dot{W}}_{\text {total }}=I_{\text {evap }} \widehat{\dot{W}}_{\text {evap }}+I_{\text {comp }+ \text { cond }} \widehat{\dot{W}}_{\text {comp }+ \text { cond }}\left(T_{\text {cond }}, T_{\text {evap }}, T_{\text {comp }, \text { in }}\right)
$$

In Eqn. (8), $I$ is the on-off status of the equipment at the current time ( 1 if on, 0 otherwise). The inputs to the power consumption virtual sensors are the temperature of the condenser return bend, temperature of the evaporator inlet and the compressor suction temperature. Neglecting the uncertainty in the readings of the current switches, the uncertainty of the estimated total power consumption is calculated by Eqn. (9).

$$
\Delta \widehat{\dot{W}}_{\text {total }}=\sqrt{\left(I_{\text {evap }} \Delta \widehat{\dot{W}}_{\text {evap }}\right)^{2}+\left(I_{\text {comp }+ \text { cond }} \Delta \widehat{\dot{W}}_{\text {comp }+ \text { cond }}\right)^{2}}
$$

Mathematical expressions for the power consumption virtual sensor outputs and their uncertainties from Cheung and Braun (2015) are listed in the Appendix for reference. 
The hourly electricity consumption of the packaged air conditioning unit was estimated by integrating the estimated total power consumption with respect to time using the trapezoidal rule as Eqn. (10).

$$
\hat{E}=\sum_{i=1}^{N-1} \frac{\left(\hat{\dot{W}}_{\text {total }, i}-\hat{\dot{W}}_{\text {total }, i+1}\right) \Delta t}{2}
$$

In Eqn. (10), $\mathrm{N}$ is the number of data points acquired during the hour and $\Delta t$ is the sampling time interval for data acquisition. Although the power consumption virtual sensors in Cheung and Braun (2015) were developed based on calibration using steady state data only, in this study it was assumed that unsteady operation of the equipment had negligible effect on integrated electricity consumption of the unit. The goal in this work was to integrate outputs of the power consumption virtual sensors to estimate total (e.g., hourly, daily, etc.) electricity consumption and unsteady operation leads to negligible errors for integrated quantities. The uncertainty of the estimated energy consumption is computed by Eqn. (11).

$$
\Delta \hat{E}=\sqrt{\left(\frac{\Delta \hat{\hat{W}}_{\text {total }, 1}}{2}\right)^{2} \Delta t+\sum_{i=2}^{N-1}\left(\Delta \widehat{\hat{W}}_{\text {total }, i}\right)^{2} \Delta t+\left(\frac{\Delta \hat{\dot{W}}_{\text {total }, N}}{2}\right)^{2} \Delta t}
$$

The uncertainty of the sampling time interval is assumed to have a negligible effect on the estimated energy consumption.

The uncertainties of the power virtual sensors that are used in Eqn. (11) are presented in the Appendix and are based on the method of Cheung and Braun (2015). They are used to calculate the average uncertainties for reliability and applicability in the algorithm.

\section{User-defined parameters of the minimization algorithm in the case study}

To use the algorithm in the case study, the length of the data acquisition period between updates and assessments of the virtual sensors, a user-defined test case for applicability assessment, thresholds for convergence indicators and a maximum data acquisition time for algorithm termination need to be specified. A two-day interval was used as the length of each short data acquisition period in this case study. A test case for assessing the virtual sensor applicability was defined by specifying somewhat arbitrary variations in the virtual sensor inputs (condenser return bend temperature, evaporator inlet temperature, compressor suction temperature and the on-off statuses of the compressor and the fans) over an expected range of values for this application. In the test case, the compressor, condenser fan and evaporator fan were assumed to be operating all the time, and Figure 3, Figure 4 and Figure 5 show the assumed variations of temperatures throughout the test case that were specified over a 100hour period for a 1-minute sampling interval. 


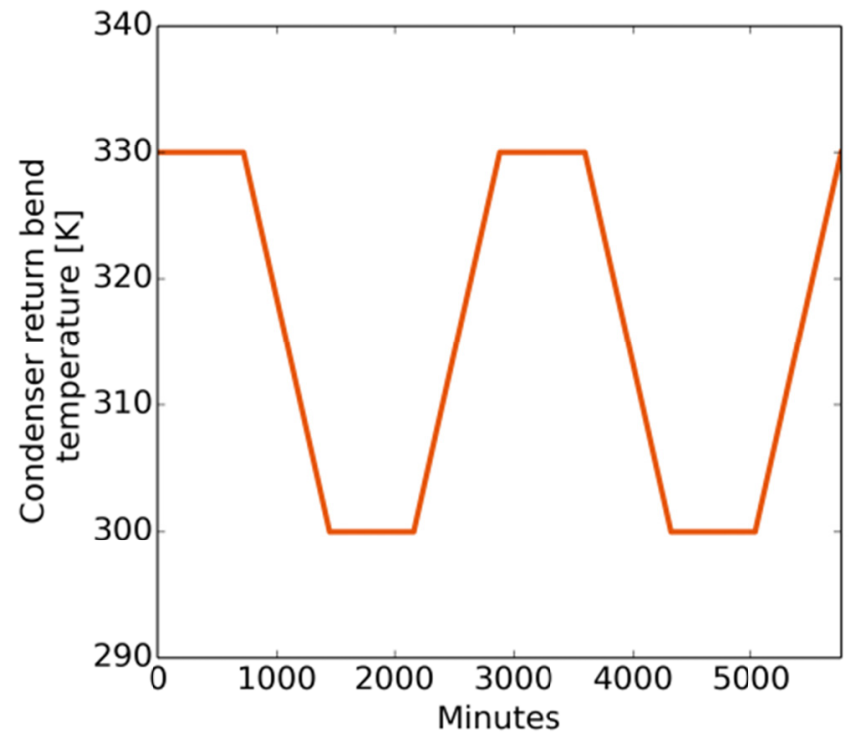

Figure 3 Time profile of condenser return bend temperature in the test case

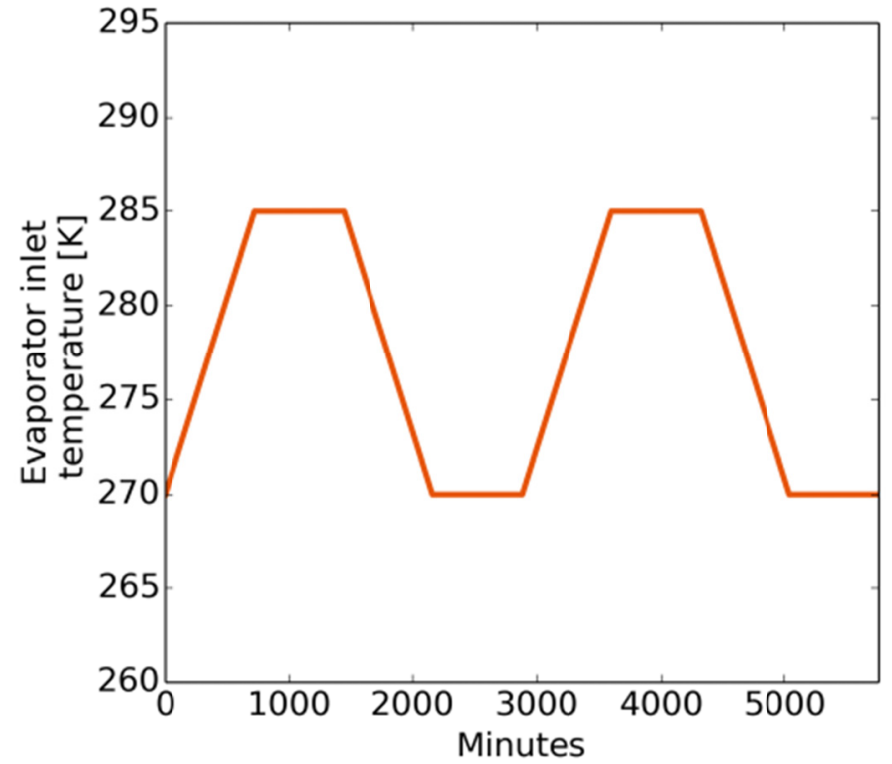

Figure 4 Time profile of evaporator inlet temperature in the test case 


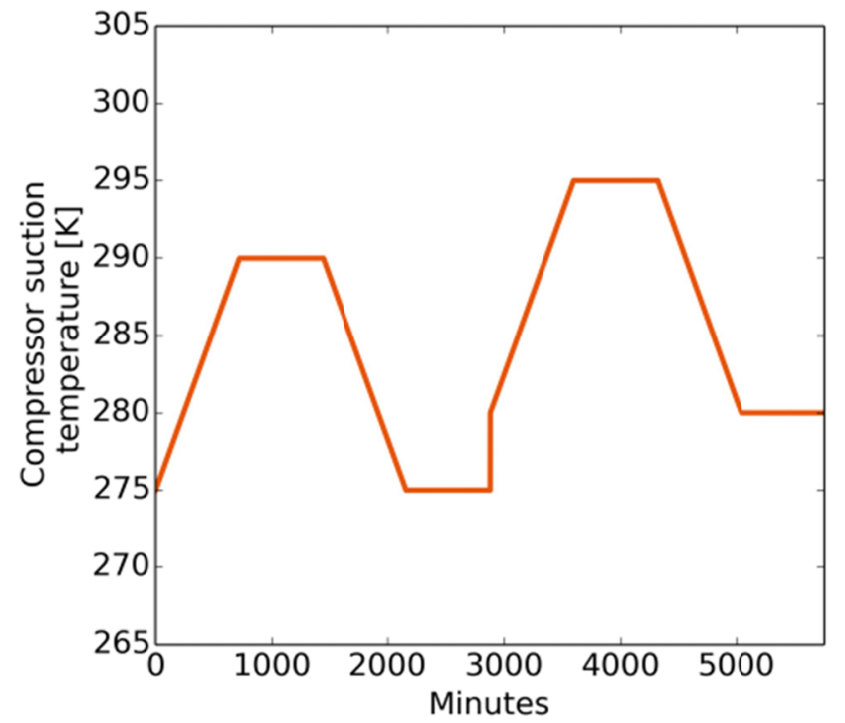

Figure 5 Time profile of compressor suction temperature in the test case

To calculate the average test case uncertainty, the virtual sensor was used to estimate the hourly energy consumption throughout the user-defined time profile with temperature values in Figure 3, Figure 4 and Figure 5. Since there are 100 hours in the time profile of the test case, 100 different hourly energy consumption were estimated in the test case after each short data acquisition period, and their uncertainties were averaged to evaluate the average test case uncertainty and the convergence indicator for applicability in Eqns. (3) and (6) to decide if the data acquisition should be stopped.

The user-defined thresholds for various indicators are tabulated in Table 2.

Table 2 Thresholds to terminate the data acquisition

\begin{tabular}{|l|l|r|}
\hline Performance indicators & Coefficient of variation & 0.03 \\
\cline { 2 - 3 } & $\begin{array}{l}\text { Average uncertainty within calibration period } \\
{[\mathrm{kWh}]}\end{array}$ & 0.02 \\
\cline { 2 - 3 } & Average uncertainty of the test case [kWh] & 0.05 \\
\hline Convergence indicators & $\operatorname{lnd}_{\mathrm{acc}}[/ \mathrm{day}]$ & 0.001 \\
\cline { 2 - 3 } & $\operatorname{lnd}_{\mathrm{rel}}[\mathrm{kWh} / \mathrm{day}]$ & 0.001 \\
\cline { 2 - 3 } & $\operatorname{lnd}_{\mathrm{app}}[\mathrm{kWh} / \mathrm{day}]$ & 0.01 \\
\hline
\end{tabular}

When all performance indicators or all convergence indicators are lower than the thresholds in Table 2, the algorithm terminates the data acquisition and process.

For demonstration purposes, the algorithm was only initiated after collecting data for 17 days and was terminated after 25 days of data regardless of other termination criteria. Another 7 
days of data were also used as validation data to illustrate the accuracy of the hourly electricity consumption virtual sensor.

\section{Computation procedure after each short data acquisition period}

At each step of the calibration process (i.e., after each short data acquisition period), the calibration data were processed to gather steady state operating data according to Cheung and Braun (2015). All of the steady-state data up to that point were then used to calibrate the two power consumption virtual sensors as described by Cheung and Braun (2015) in order to build the hourly electricity consumption sensor using Eqns. (9) and (10) for each air conditioner. The hourly electricity consumption sensors estimate the electricity consumption of the air conditioners over every hour, including non-steady operating hours. The estimates were used to compute the performance and convergence indicators to evaluate whether the data acquisition should continue. While the method was designed to be implemented within any software language or platform, the computation procedure was carried out with Python 2.7 scripts in this case study.

\section{Results and Discussion}

The calibration results after the end of the automated calibration algorithm are tabulated in Table 3 and Table 4.

Table 3 Number of days required to collect enough data for calibrating the virtual sensors by the minimization algorithm

\begin{tabular}{|c|c|c|}
\hline Unit & $\begin{array}{c}\text { Number of } \\
\text { days }\end{array}$ & Termination criteria \\
\hline RTU 2 & 19 & $\begin{array}{r}\text { User requirements for sensor performance in terms of } \\
\text { accuracy, reliability and applicability are met, and extra data } \\
\text { is unlikely to improve the sensor performance. }\end{array}$ \\
\hline RTU 3 & 23 & Extra data is unlikely to improve the sensor performance. \\
\hline RTU 4 & 25 & The collection period has exceeded an upper limit. \\
\hline
\end{tabular}

Table 3 shows that the algorithm was terminated for different reasons for the three air conditioners. To examine the accuracy of the hourly electricity consumption sensors, the data collected for 15 days in September are used for validation only, and the predicted and measured energy consumption are plotted for the calibration and validation periods for the different units in Figure 6, Figure 7 and Figure 8. 


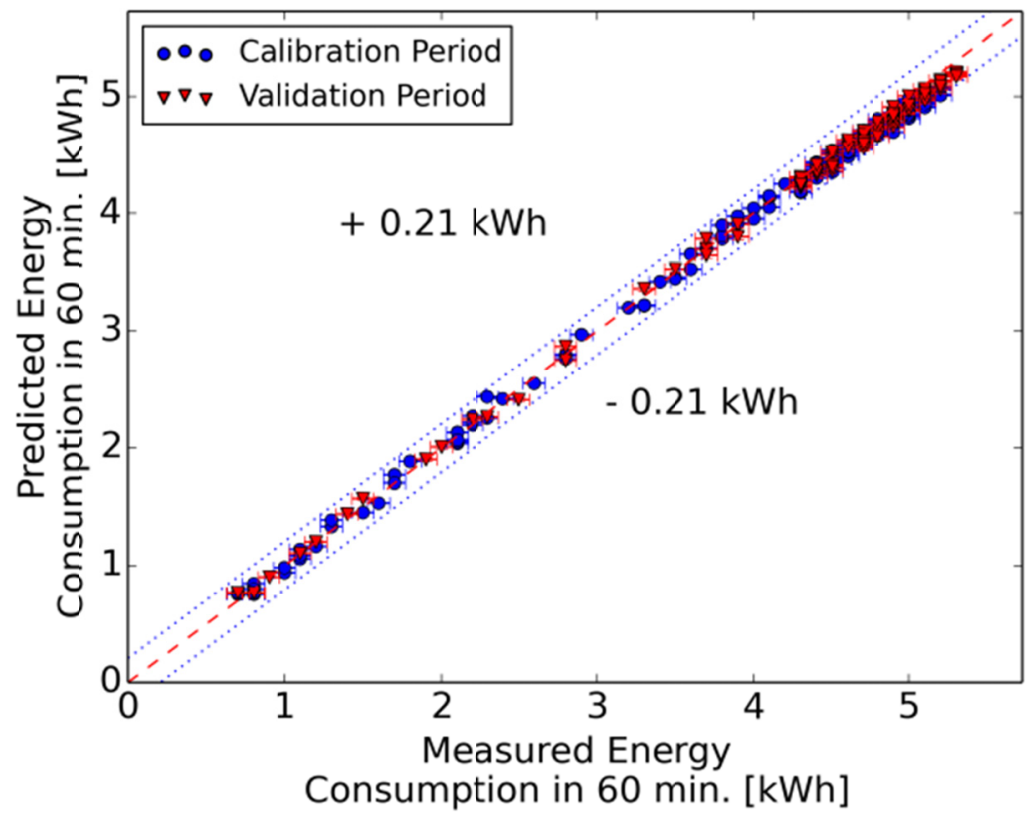

Figure 6 Comparison between the measured and estimated hourly electricity consumption of RTU 2

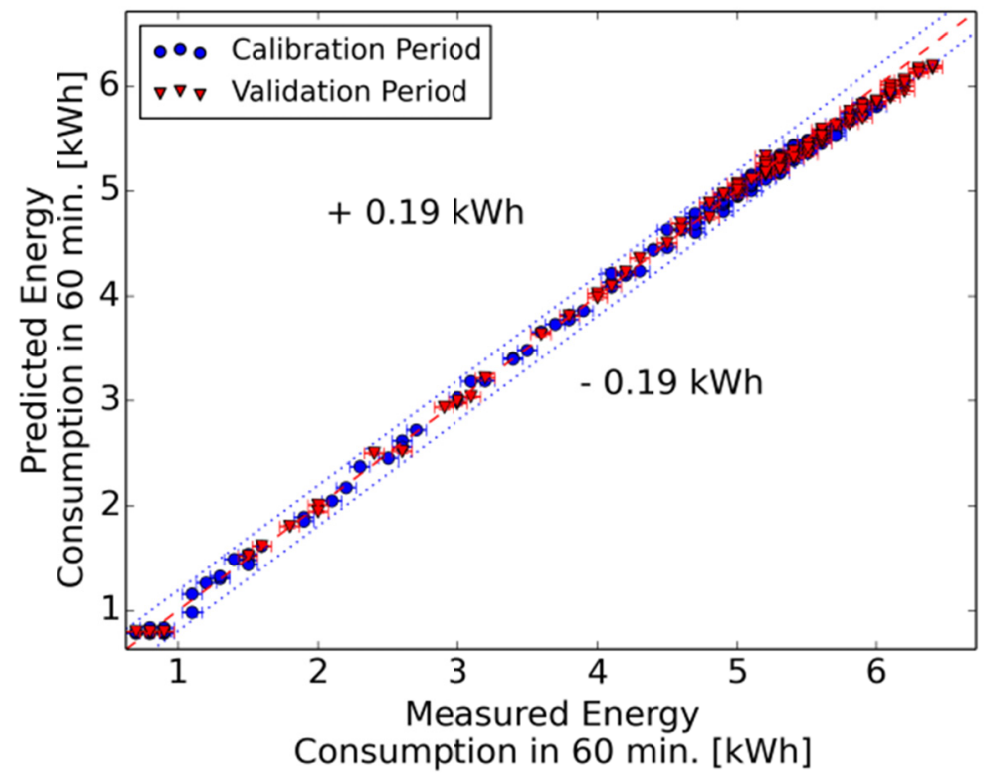

Figure 7 Comparison between the measured and estimated hourly electricity consumption of RTU 3 


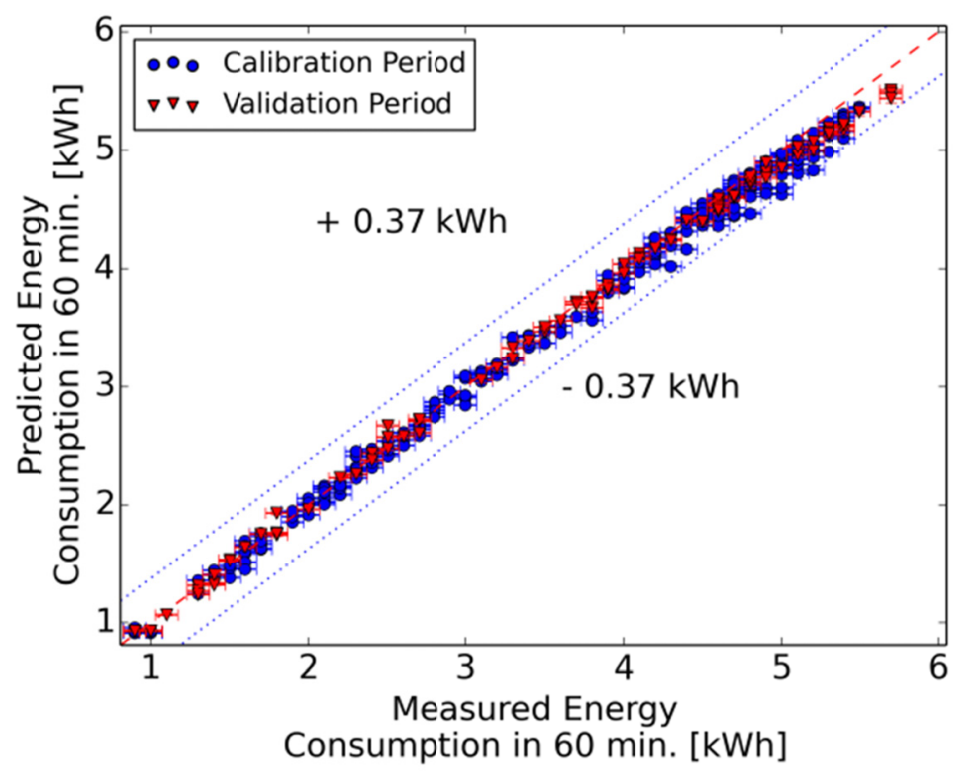

Figure 8 Comparison between the measured and estimated hourly electricity consumption of RTU 4

Figure 6, Figure 7 and Figure 8 show that all virtual sensors have acceptable accuracy for both the calibration and validation scenarios with a maximum deviation at $0.37 \mathrm{kWh}$. They also show that the virtual sensor for RTU 3 is the most accurate sensor among the three systems because the maximum deviation in Figure 7 is the smallest. To understand how the calculation of the performance and convergence indicators lead to the decisions in Table 3, the performance and convergence indicators at the termination of data acquisition in Table 4 were studied.

Table 4 The performance indicators, the convergence indicators, and the length of data acquisition at termination of data acquisition for different units

\begin{tabular}{|l|c|c|c|}
\hline Unit & RTU 2 & RTU 3 & RTU 4 \\
\hline Coefficient of variation & 0.0256 & 0.0201 & 0.0363 \\
\hline $\begin{array}{l}\text { Average uncertainty within calibration period } \\
{[\mathrm{kWh}]}\end{array}$ & 0.0160 & 0.0160 & 0.0141 \\
\hline Average uncertainty of the test case [kWh] & 0.0425 & 0.0570 & 1.46 \\
\hline Ind $_{\mathrm{c}}[/$ day] & 0.000539 & 0.000647 & 0.00155 \\
\hline Ind $_{\text {cal }}[\mathrm{kWh} /$ day] & 0.000677 & 0.000100 & 0.000211 \\
\hline Ind $_{\text {test }}[\mathrm{kWh/day]}$ & 0.00883 & 0.00320 & 0.0944 \\
\hline Length of data acquisition & 19 & 23 & 25 \\
\hline
\end{tabular}

Table 4 shows that the coefficient of variation, the average uncertainty within the calibration period and the average uncertainty of the test case of RTU 2 virtual sensor are all smaller than the thresholds in Table 2. That's why the algorithm determined that the RTU 2 virtual sensor satisfied the user requirement on sensor accuracy, reliability and applicability and stopped the data collection on the $19^{\text {th }}$ day. Although the average uncertainty within the calibration period 
of the virtual sensors of RTU 3 and 4 are smaller than the thresholds in Table 2, their average uncertainties for the test case are not. Therefore, the RTU 3 and 4 virtual sensors did not satisfy the user applicability requirement, and data acquisition was not terminated for this reason.

Although the RTU 3 virtual sensor performance did not satisfy the user requirement on the $23^{\text {rd }}$ day, all of its convergence indicators in Table 4 are smaller than their thresholds. This implies that the accuracy, reliability and applicability of the RTU 3 virtual sensor could not be improved by extra data and its data acquisition was stopped on the $23^{\text {rd }}$ day. A similar situation occurred for the RTU 2 sensor on the $19^{\text {th }}$ day and its data acquisition was terminated for the same reason. However, the convergence indicators of the RTU 4 sensor were higher than the thresholds on the $25^{\text {th }}$ day and hence its data collection was not terminated by this criterion. To examine if the calculation of convergence indicators can truly predict the significance of extra data on the calibration process, the performance indicators of calibration results generated by using 25 days of RTU 3 data were studied as shown in Table 5.

Table 5 Calibration results of RTU 3 virtual sensor under different data acquisition time

\begin{tabular}{|l|c|c|}
\hline Unit & RTU 3 & RTU 3 \\
\hline Coefficient of variation & 0.0201 & 0.0209 \\
\hline $\begin{array}{l}\text { Average uncertainty within calibration period } \\
\text { [kWh] }\end{array}$ & 0.0160 & 0.0158 \\
\hline Average uncertainty of the test case [kWh] & 0.0570 & 0.052 \\
\hline Length of data acquisition & 23 & 25 \\
\hline
\end{tabular}

Table 5 shows that only the average uncertainty of the test case was reduced significantly (by 9.1\%) when the data acquisition period was increased from 23 to 25 days. However, when the change is calculated as a percentage of the minimum hourly energy consumption estimated in the test case, it is only around $0.1 \%$. This shows that 23 days of data was sufficient for calibrating the virtual sensor.

The RTU 4 data acquisition was stopped on the $25^{\text {th }}$ day because it reached the maximum data collection period limit even though the final average uncertainty and the convergence indicators for the test case in Table 4 are significantly above the thresholds in Table 2 . Virtual sensor outputs and uncertainties of the test case are shown in Figure 9. 


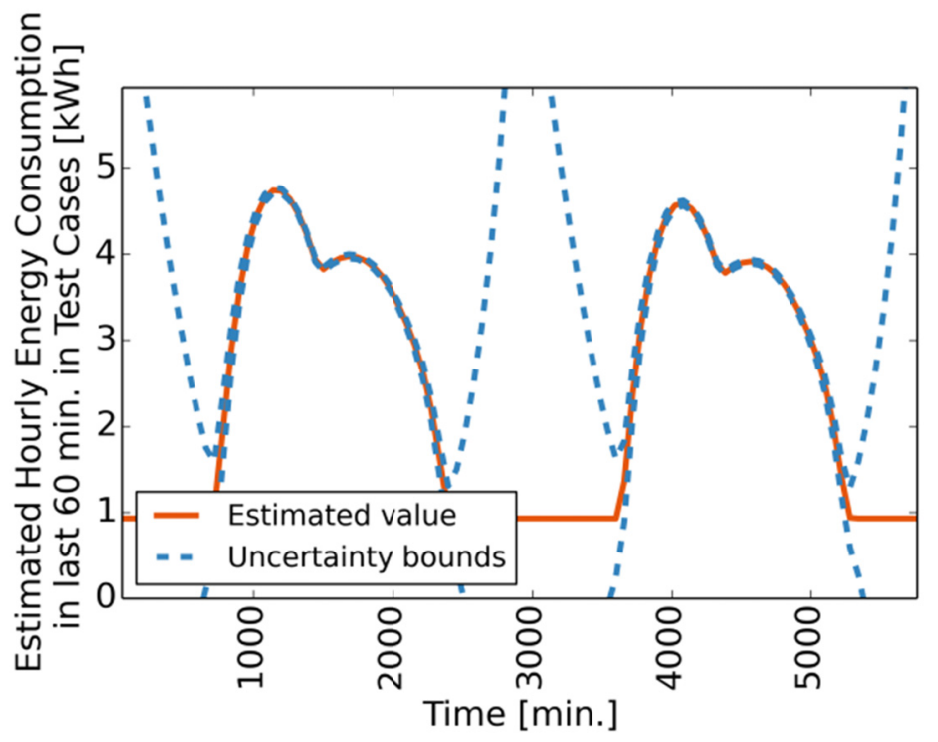

Figure 9 Estimated hourly electricity consumption of RTU 4 after calibrating its virtual sensor with 25 days of data

Comparisons of Figure 3 and Figure 9 indicate that the virtual sensor of RTU 4 cannot estimate hourly electricity consumption reliably whenever the condenser return bend temperature of RTU 4 approaches $330 \mathrm{~K}$. At this condition, the virtual sensor estimates the hourly electricity consumption to be $1 \mathrm{kWh}$ and the uncertainty bounds become large positive and negative values. At this condition, the compressor and condenser fan power consumption was estimated to be $0 \mathrm{~kW}$, whereas the evaporator fan power use was $1 \mathrm{~kW}$. The large uncertainty bounds and $0 \mathrm{~kW}$ compressor and condenser fan power consumption do not occur for the estimates of the hourly electricity consumption of RTU 2 and 3 as shown in Figure 10 and Figure 11.

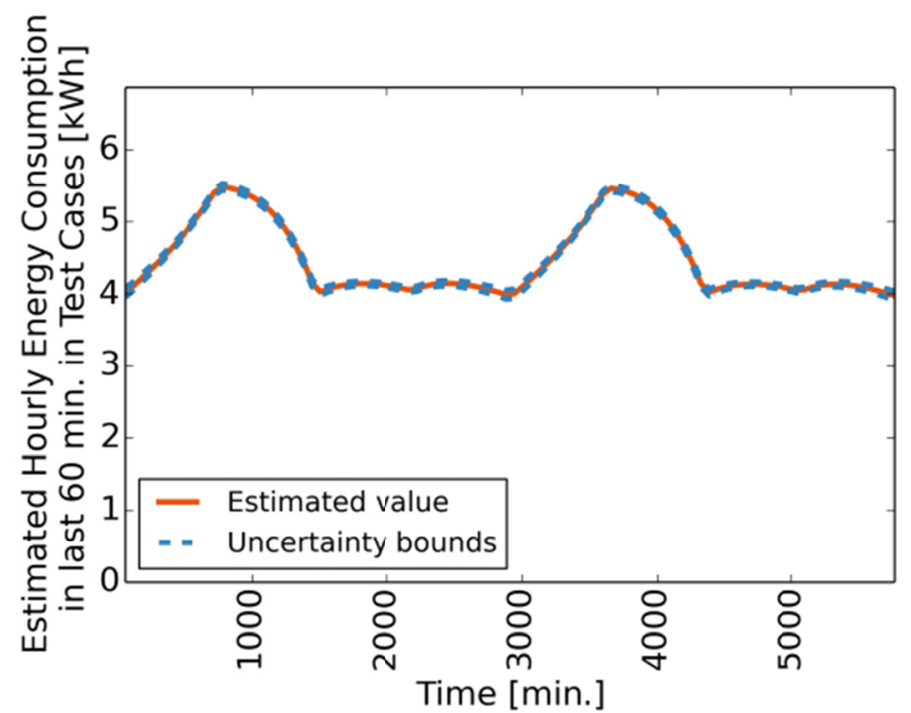

Figure 10 Estimated hourly electricity consumption of RTU 2 after calibrating its virtual sensor with 19 days of data 


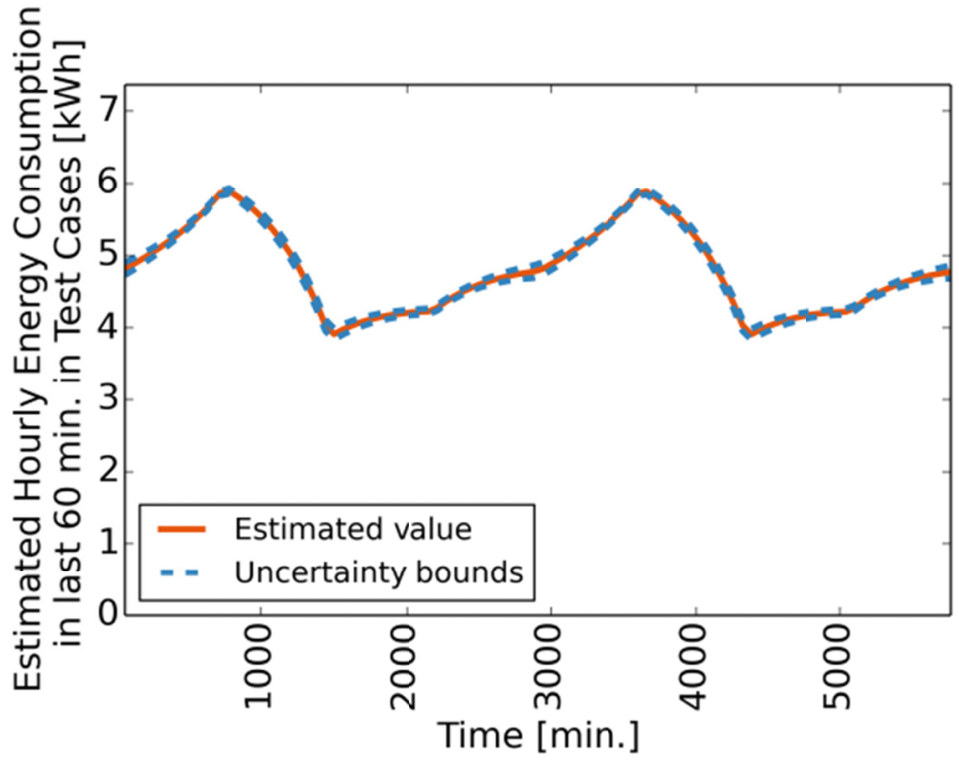

Figure 11 Estimated hourly electricity consumption of RTU 3 after calibrating its virtual sensor with 23 days of data

Figure 10 and Figure 11 show that the virtual sensors for RTU 2 and RTU 3 estimate positive hourly electricity consumption with negligible uncertainty for all the test case conditions including when the condenser return bend temperature approaches 330K. The ranges of condenser return bend temperature in the calibration data of the virtual sensors are compared for the three units in Table 6.

Table 6 Condenser return bend temperature in the calibration data of the virtual sensors at the end of their calibration period

\begin{tabular}{|c|c|}
\hline Unit & Range of condenser return bend temperature [K] \\
\hline RTU 2 & $304.4-324.3$ \\
\hline RTU 3 & $312.6-335.2$ \\
\hline RTU 4 & $301.5-318.3$ \\
\hline
\end{tabular}

Table 6 shows that the range of condenser return bend temperature in the calibration data of RTU 4 is lower than that of RTU 2 and 3. Further investigation found that the second highest condenser return bend temperature in the RTU 4 sensor calibration data is only around $315.3 \mathrm{~K}$, compared to $323.9 \mathrm{~K}$ for RTU 2. The lack of data at higher condensing temperatures means that the virtual sensor could not learn the correct behavior over the intended range of applicability. The convergence indicator for the RTU 4 test case in Table 4 is also higher than thresholds in Table 2 because the calibration data set was still changing significantly before the data acquisition was stopped. 


\section{Conclusions}

This paper presented an algorithm that minimizes the data collection period for calibrating a virtual sensor that meets user specified requirements when the data is collected during normal operation of an air conditioning unit. The algorithm updates the calibration at user specified intervals until criteria for accuracy, reliability and applicability of the sensor are met or if a maximum data collection period is reached. The algorithm was demonstrated for virtual sensors that output hourly electricity consumption for 3 packaged air conditioners operating at an existing small commercial building. The collection periods needed and stopping criteria were different for the 3 units but the results demonstrated the major features of the algorithm that lead to accurate virtual sensors.

\section{Acknowledgements}

The authors would like to acknowledge the sponsorship of the Consortium for Building Energy Innovation of the Department of Energy, Hugh Henderson for sensor installation on the packaged air conditioners and Andrew Hjortland for his collaboration on the data management for multiple projects related to the data.

\section{References}

ASHRAE. (2014). ASHRAE Guideline 14-2014: Measurement of Energy, Demand, and Water Savings. Atlanta: American Society of Heating, Refrigerating and Air-Conditioning Engineers, Inc.

Cheung, H., \& Braun, J. E. (2016). A general method for calculating the uncertainty of virtual sensors for packaged air conditioners. International Journal of Refrigeration, 63, 225 -236.

John, R. C., \& Draper, N. R. (1975). D-Optimality for Regression Designs: A Review. Technometrics, 17(1), 15-23.

Jones, P. G. (2011). Optimal Design of Experiments: A Case Study Approach. Wiley.

Kim, W., \& Braun, J. E. (2015). Extension of a virtual refrigerant charge sensor. International Journal of Refrigeration, 55, 224-235.

Kusiak, A., Li, M., \& Zheng, H. (2010). Virtual models of indoor-air-quality sensors. Applied Energy, 87, 2087 - 2094.

Li, H., Yu, D., \& Braun, J. E. (2011). A review of virtual sensing technology and application in building systems. HVAC\&R Research, 17(5), 619-645.

Mckay, M. D., Beckman, R. J., \& Conover, W. J. (2000). A Comparison of Three Methods for Selecting Values of Input Variables in the Analysis of Output from a Computer Code. Technometrics, 42(1), 55-61.

Montgomery, D. (2005). Design and Analysis of Experiments, 6th Edition. Jon Wiley \& Sons . 
Nocedal, J., \& Wright, S. (2006). Numerical optimization. Springer.

Reddy, T. A., \& Claridge, D. E. (2000). Uncertainty of "Measured" Energy Savings from Statistical Baseline Models. HVAC\&R Research, 6(1), 3-20.

Song, L., Wang, G., \& Brambley, M. R. (2013). Uncertainty analysis for a virtual flow meter using an air-handling unit chilled water valve. HVAC\&R Research, 19(3), 335-345.

Taguchi, G., Chowdhury, S., \& Wu, Y. (2004). Taguchi's Quality Engineering Handbook. Wiley.

Vasudevan, J. (2015). Training and Evaluation of Virtual Sensors for Rooftop Units. Master thesis. West Lafayette: Purdue University.

Wang, G., Song, L., Andiroglu, E., \& Shim, G. (2014). Investigations on a virtual airflow meter using projected motor and fan efficiencies. HVAC\&R Research, 20(2), 178-187.

Yu, D., Li, H., \& Yang, M. (2011). A virtual supply airflow rate meter for rooftop air-conditioning units. Building and Environment, 46, 1292-1302.

Yu, Y., \& Li, H. (2015). Virtual in-situ calibration method in building systems. Automation in construction, 59, 59-67.

Zhao, X., Yang, M., \& Li., H. (2012). A virtual condenser fouling sensor for chillers. Energy and Buildings, 52, 68-76. 


\section{Appendix: Mathematical expressions of virtual sensor output and uncertainties}

This appendix presents the virtual sensor output and uncertainty relations from Cheung and Braun (2015) that were employed in the case study.

\section{Virtual sensor for evaporator fan power consumption}

This simple virtual sensor was created by averaging the power consumption of the evaporator fan during steady state fan-only operation and the result is presented in Eqn. (12).

$$
\widehat{\dot{W}}_{\text {evap }}=\overline{\dot{W}}_{\text {evap }, \text { cal }}
$$

The uncertainty of the virtual sensor output is given by Eqn. (13).

$$
\begin{aligned}
& \Delta \widehat{\dot{W}}_{\text {evap }} \\
& =\sqrt{\frac{\sum_{i}\left(\Delta \dot{W}_{\text {evap }, \text { train }, i}\right)^{2}}{n^{2}}+\left(1+\frac{1}{n^{2}}\right)\left(t_{n-1,0.95} \sigma_{\dot{W}_{\text {evap }}}\right)^{2}+\left(\frac{\widehat{\dot{W}}_{\text {evap }}}{n} \sum_{i} \frac{\Delta \dot{W}_{\text {evap }, \text { train }, i}}{\dot{W}_{\text {evap }, \text { train }, i}}\right)^{2}}
\end{aligned}
$$

The uncertainty Eqn. (13) depends on the amount of the calibration data and the measurement uncertainties. A large set of calibration data with small measurement uncertainty leads to a small uncertainty of the virtual sensor estimation.

Virtual sensor for power consumption of compressor and condenser fan The virtual sensor for the compressor and condenser fan power consumption is given with Eqn. (14) .

$$
\begin{aligned}
& \dot{W}_{\text {comp }+ \text { cond }} \\
& =\frac{\kappa P_{v}\left(T_{\text {evap }}\right)}{\kappa-1} \frac{\left[\beta_{\text {comp }, 1}-\beta_{\text {comp }, 2}\left(\left(\frac{P_{v}\left(T_{\text {cond }}\right)}{P_{v}\left(T_{\text {evap }}\right)}\right)^{\frac{1}{\kappa}}-1\right)\right]\left(\left(\frac{P_{v}\left(T_{\text {cond }}\right)}{P_{v}\left(T_{\text {evap }}\right)}\right)^{\left(\frac{\kappa-1}{\kappa}\right)}-1\right)}{\left(1+\beta_{\text {comp }, 3}\left(P_{v}\left(T_{\text {evap }}\right)-\beta_{\text {comp }, 4}\right)\right)\left(1+\beta_{\text {comp }, 5}\left(P_{v}\left(T_{\text {cond }}\right)-\beta_{\text {comp }, 6}\right)\right)}
\end{aligned}
$$

where,

$$
\kappa=\frac{c_{p}\left(P=P_{v}\left(T_{\text {evap }}\right), T=T_{\text {comp }, \text { in }}\right)}{c_{v}\left(P=P_{v}\left(T_{\text {evap }}\right), T=T_{\text {comp }, \text { in }}\right)}
$$

The uncertainty of the virtual sensor output is determined using Eqns. (16), (17), (18), (19) and (20). 


$$
\begin{aligned}
& \left(\Delta \widehat{\dot{W}}_{\text {comp }+ \text { cond }}\left(\vec{x}, \overrightarrow{\hat{\beta}}_{\text {comp }}\right)\right)^{2} \\
& =\sum_{i} \sum_{k}\left(\left(\sum_{p} \frac{\partial \hat{\dot{W}}_{\text {comp }+ \text { cond }}\left(\vec{x}, \overrightarrow{\hat{\beta}}_{\text {comp }}\right)}{\partial \overrightarrow{\hat{\beta}}_{\text {comp }, p}} \frac{\partial \overrightarrow{\hat{\beta}}_{\text {comp }, p}}{\partial \vec{x}_{\text {train }, l k}}\right) \Delta x_{\text {train }, l k}\right)^{2} \\
& +\sum_{k}\left(\left(\sum_{p} \frac{\partial \hat{\dot{W}}_{\text {comp }+ \text { cond }}\left(\vec{x}, \overrightarrow{\hat{\beta}}_{c o m p}\right)}{\partial \overrightarrow{\hat{\beta}}_{\text {comp }, p}} \frac{\partial \overrightarrow{\hat{\beta}}_{\text {comp }, p}}{\partial \dot{W}_{\text {comp }+ \text { cond,train }, k}}\right) \Delta \dot{W}_{\text {comp }+ \text { cond }, \text { train }, k}\right)^{2} \\
& +t_{n-m, 0.95}^{2} \sigma_{w x}^{2} \\
& +t_{n-m, 0.95}^{2} \sigma_{w x}^{2}\left(( \frac { \partial \hat { \hat { W } } _ { \text { comp } + \text { cond } } ( \vec { x } , \vec { \hat { \beta } } _ { c o m p } ) } { \partial \vec { \hat { \beta } } _ { c o m p } } ) ^ { T } \left(\boldsymbol{J}_{w, \text { train }}^{T} \boldsymbol{J}_{w, \text { train }}\right.\right. \\
& \left.\left.-\left[\overrightarrow{\dot{W}}_{\text {comp }+ \text { cond,train }}-\overrightarrow{\hat{\dot{W}}}_{\text {comp }+ \text { cond,train }}\right]\left[\boldsymbol{H}_{w, \text { train }}\right]\right)^{-1}\left(\frac{\partial \hat{\hat{W}}_{\text {comp }+ \text { cond }}\left(\vec{x}, \overrightarrow{\hat{\beta}}_{\text {comp }}\right)}{\partial \overrightarrow{\hat{\beta}}_{\text {comp }}}\right)\right) \\
& +\left(\frac{\widehat{\dot{W}}_{\text {comp }+ \text { cond }}\left(\vec{x}, \overrightarrow{\hat{\beta}}_{\text {comp }}\right)}{n} \sum_{i} \frac{\Delta \dot{W}_{\text {comp }+ \text { cond,train }, i}}{\dot{W}_{\text {comp }+ \text { cond } \text { train } . i}}\right)^{2} \\
& +\sum_{i}\left(\frac{\partial \hat{\hat{W}}_{\text {comp }+ \text { cond }}\left(\vec{x}, \overrightarrow{\hat{\beta}}_{\text {comp }}\right)}{\partial x_{i}} \Delta x_{i}\right)^{2}
\end{aligned}
$$

$$
\sigma_{w x}^{2}=\frac{\sum_{i}\left(\hat{\dot{W}}_{\text {comp }+ \text { cond }, \text { train }, i}\left(\vec{x}_{\text {train }, i}, \overrightarrow{\hat{\beta}}_{\text {comp }}\right)-\dot{W}_{\text {comp }+ \text { cond }, \text { train }, i}\right)^{2}}{n-m}
$$


The t-statistics in Eqn. (16) depend on the amount of calibration data with estimation uncertainty decreasing with increasing data. The magnitude of the expression with the Jacobian and the Hessian matrices in Eqn. (16) depends on applicability of the virtual sensor to the current input, and it grows as the virtual sensor inputs differ from the calibration data points. 